\title{
Gaussian approximation for Ising model. Variational approach
}

\author{
V.S.Yanishevsky \\ Ivan Franko Drogobych State Pedagogical University, \\ 24 I.Franko Str., 293720 Drogobych, Lviv Region, Ukraine \\ Received December 25, 1998, in final form May 3, 1999
}

\begin{abstract}
A variant of a Gaussian approximation in Ising model of phase transition is suggested. The method of functional integration with the application of a variational method is put in the basis of the approach. The Hamiltonian (the functional) is presented which in the method of functional integration produces the known results of a two-tails approximation obtained by a diagram method. The application of a variational method eliminates a principal demerit of the two-tails approximation - incorrect description of the phase transition order. The results of numerical calculations for two and threedimensional lattices are presented.
\end{abstract}

Key words: Ising model, functional integration, Gaussian approximation

PACS: $75.10 . \mathrm{H}$

\section{Introduction}

In this paper the self-consistent method of inclusion of the space fluctuations of the order parameter in Ising type models of phase transitions is considered. As it is known the number of exactly solved models of phase transitions is rather restricted, therefore the approximate methods of investigation are widely used. In particular a rather simple and effective way of the phase transitions description is the mean field theory which is based on introducing the homogeneous order parameters for the whole system. The next step is the problem of including the order parameter fluctuations. One of the widely-applied methods is the diagram technique for the spin operators [1-4] which permits to make the perturbations theory expansions for the spin correlation functions and thermodynamic values according to the corresponding expansion parameters (e.g. $\sim 1 / r_{0}^{d}, r_{0}$ is the exchange interaction radius, $d$ is the dimension of system, or by $\sim 1 / z$, where $z$ is the coordination number of the lattice). The first approximation by these parameters is the so-called one-loop approximation or otherwise - the random phase approximation. More complex approximations are obtained by summation a certain class of diagrams. In particular, a two-tails approximation is known $[1,4,5]$ for a spin moment and for a spin correlation 
function in which the order parameter fluctuation around the mean field is described by Gaussian distribution with the variance determined by two-tails. But one of the shortcomings of the mentioned approximations is that at the self-consistent method including the mean field and fluctuations, the ruptures of the order parameter temperature dependence arise which formally can be considered as the first order phase transition. A number of methods with the purpose of removing these shortcomings is known too [6-8]. In [6] the Brillouin zone (for the primitive cubic lattice) is modified for this purpose, in [7] a certain number of two-tails is limited. A more consequent method is considered in [8], where the necessity is pointed out for taking into consideration the additional series of diagrams which have the same order of quantity as the two-tails approximation diagrams. For this purpose the author of [8] considered the system of equations for spin cumulants and suggested the splitting way of the 4-th order spin cumulant for getting the closed system of equations for the spin cumulants up to the 3-rd order. Though the author showed that in the vicinity of the critical point the method led to the second order phase transition, the dependence of the order parameter in the wide temperature range is not given which seems to be connected with the numerical analysis complicity of the obtained system of equations.

Taking into consideration the above mentioned facts, a rather simple and consecutive way of improving two-tails approximation results is proposed. The method of functional integration with the variational method is used. First, a two-tails approximation in the functional approach is obtained and later the Hamiltonian of two-tails approximation is used as a trial in the variational method. Numerical results are given for the two- and three-dimensional Ising spin model $S=1 / 2$ on the square and cubic lattices where the nearest neighbours interact respectively.

\section{The two-tails approximation in the functional integration method}

Let's consider Hamiltonian of Ising model in interaction between the nearest neighbours

$$
H=-\sum_{<i j>} I \sigma_{i} \sigma_{j}-\sum_{i} h \sigma_{i}
$$

Having introduced, in a traditional way, the Fourier-transformation of the spin operators $\sigma_{\mathbf{k}}=\frac{1}{\sqrt{N}} \sum_{i} \sigma_{i} \mathrm{e}^{\mathbf{i k r}}$, we can write this Hamiltonian as follows:

$$
H=-\frac{1}{2} \sum_{\mathbf{k}} I_{\mathbf{k}} \sigma_{\mathbf{k}} \sigma_{-\mathbf{k}}-\sum_{i} h \sigma_{i} .
$$

Here $I_{\mathbf{k}}=z I \gamma_{\mathbf{k}}, \gamma_{\mathbf{k}}=\frac{1}{z} \sum_{\delta} e^{i \mathbf{k} \delta}$, summing over $\mathbf{k}$ is carried out within the limits of the first Brillouin zone. The vector $\boldsymbol{\delta}$ includes the nearest neighbours in the spin lattice.

Functional integration methods are widely used in the phase transition investigations and a lot of literature is devoted to them (e.g., $[1,2,4,9-14])$. In the present 
paper the known representation of partition functions is put into the base of the functional method.

$$
Z=\int(\mathrm{d} \rho) \mathrm{e}^{-\frac{1}{2} \sum_{\mathbf{k}} \rho_{\mathbf{k}} \rho_{-\mathbf{k}}} \mathrm{e}^{-\beta \sum_{i} F_{i d}\left(\beta h+h_{i}\right)} / \int(\mathrm{d} \rho) \mathrm{e}^{-\frac{1}{2} \sum_{\mathbf{k}} \rho_{\mathbf{k}} \rho_{-\mathbf{k}}}
$$

where $\beta F_{i d}=-\ln Z_{i d}, Z_{i d}=\mathrm{Sp} \mathrm{e}^{\left(\beta h+h_{i}\right) \sigma_{i}}$ - means the partition function of the spin in the fluctuation field $h_{i}=\frac{1}{\sqrt{N}} \sum_{\mathbf{k}} \sqrt{\beta I_{\mathbf{k}}} \rho_{\mathbf{k}} \mathrm{e}^{\mathrm{ikr}}$. For the spin with the value of $S$ the partition function in the external field is

$$
Z_{i d}=\operatorname{sh}[(S+1 / 2) y] / \operatorname{sh}(1 / 2 y), \quad y=\beta h+h_{i} .
$$

In a standard procedure we can introduce the mean field [12], which is connected with the Fourier component $\rho_{0}: h_{i}=h_{0}+h_{i}^{\prime}, h_{0}=\frac{1}{\sqrt{N}} \sqrt{\beta I_{0}} \rho_{0}, h_{i}^{\prime}=\frac{1}{\sqrt{N}} \sum_{\mathbf{k} \neq 0} \sqrt{\beta I_{\mathbf{k}}} \rho_{\mathbf{k}} \mathrm{e}^{\mathbf{i k r}}$ and after replacing $\rho_{0} \rightarrow \sqrt{N} \rho_{0}$ in the thermodynamic limit we present the partition function as

$$
Z=Z_{0} \int(\mathrm{d} \rho)^{\prime} \mathrm{e}^{-\beta H^{\prime}(\rho)} / \int(\mathrm{d} \rho)^{\prime} \mathrm{e}^{-\frac{1}{2} \sum_{\mathbf{k} \neq 0} \rho_{\mathbf{k}} \rho_{-\mathbf{k}}} .
$$

Here $Z_{0}=\mathrm{e}^{-\beta F\left(\bar{\rho}_{0}\right)}, F\left(\bar{\rho}_{0}\right)=\frac{N}{2} I_{0} \bar{\rho}_{0}^{2}+N F_{i d}\left(h_{\mathrm{M}}\right)$ is a free energy in the mean field approximation, and

$$
H^{\prime}(\rho)=\frac{1}{2 \beta} \sum_{\mathbf{k} \neq 0} \rho_{\mathbf{k}} \rho_{-\mathbf{k}}+\sum_{i}\left[F_{i d}\left(h_{\mathrm{M}}+h_{i}^{\prime}\right)-F_{i d}\left(h_{\mathrm{M}}\right)\right]
$$

has a sense of fluctuations component of the system Hamiltonian and is a functional of the $\rho_{\mathbf{k}}(\mathbf{k} \neq 0)$ variables that describe the order parameter fluctuations; $h_{\mathrm{M}}=$ $h+I_{0} \bar{\rho}_{0}$ is a mean field that acted on the spin. The consideration of the corrections to the mean field approximation is done by means of the expansion by powers of $h_{i}^{\prime}$

$$
\sum_{i} F_{i d}\left(h_{\mathrm{M}}+h_{i}^{\prime}\right)=\sum_{i, n} \frac{1}{n !} F_{i d}^{(n)}\left(h_{\mathrm{M}}\right)\left(h_{i}^{\prime}\right)^{n},
$$

and after summing over the particles number we can get

$$
\sum_{i}\left(h_{i}^{\prime}\right)^{n}=\sum_{\mathbf{k}}(\sqrt{N})^{-n} \sqrt{\beta I_{\mathbf{k}_{1}}} \cdot \ldots \cdot \sqrt{\beta I_{\mathbf{k}_{n}}} \rho_{\mathbf{k}_{1}} \cdot \ldots \cdot \rho_{\mathbf{k}_{n}} \boldsymbol{\delta}\left(\mathbf{k}_{1}+\ldots+\mathbf{k}_{n}\right)
$$

here $\boldsymbol{\delta}\left(\mathbf{k}_{1}+\ldots+\mathbf{k}_{n}\right)$ is a Kronecker symbol, and the sum of wave vectors is closed: $\mathbf{k}_{1}+\ldots+\mathbf{k}_{n}=0$. The summation over vectors $\mathbf{k}_{1}, \ldots, \mathbf{k}_{n}$ in (2.7) will be carried out independently, otherwise, according to $[9,13]$, the sums are reduced. Traditionally contribution of $\sim \frac{1}{N} \sum_{\mathbf{k} \neq 0} \beta I_{\mathbf{k}} \rho_{\mathbf{k}} \rho_{-\mathbf{k}}$ is included in zeroth-order approximation, that will result in a random phase approximation, and the following components of a series are considered using a perturbation theory.

Let's also mark that the representation of the partition function in the form of a functional integral such as (2.5)-(2.7) has played a substantial role in constructing the microscopic theory of phase transition [9-13]. It is proved that for describing the 
fluctuations of an order parameter of three-dimensional systems in the neighbourhood of a critical point it is necessary to apply a basis distribution, which includes summands with $n \leqslant 6$ in (2.7). With the help of a specially designed method of a renormalization group for calculating a N-multiple integral of a non-Gaussian type the quantitative description of phase transition of the first and second orders in the neighbourhood of a critical point was obtained. In the present paper, certainly, we do not touch upon this special area of the theory of phase transition, but we use the above evocative representation for constructing a self-consistent approximation.

One can execute a certain selective summation of components of a series (2.7) in a Hamiltonian (2.6), including the corresponding result in zeroth-order approximation. The indicated procedure is easily realized for summands such as

$$
\sim\left(\frac{1}{N} \sum_{\mathbf{k} \neq 0} \beta I_{\mathbf{k}} \rho_{\mathbf{k}} \rho_{-\mathbf{k}}\right)^{n}
$$

The summation of such series, as will be shown below, is equivalent to a two-tails approximation in a diagram method. The simplest way of executing the above mentioned summation is the application of the expansion on the irreducible sums of vectors $\mathbf{k}_{1}, \ldots, \mathbf{k}_{n}$. For this purpose such an identical transformation is used

$$
\sum_{i} F_{i d}\left(h_{\mathrm{M}}+h_{i}^{\prime}\right)=N \frac{1}{N} \sum_{i} \mathrm{e}^{h_{i}^{\prime} \partial / \partial(\beta h)} F_{i d}\left(h_{\mathrm{M}}\right),
$$

and an action of a differential operator will be written as follows:

$$
\begin{aligned}
\frac{1}{N} \sum_{i} e^{h_{i}^{\prime} \partial / \partial(\beta h)=} & \exp \left(\sum_{\mathbf{k}} \frac{1}{n !}(\sqrt{N})^{-n} \sqrt{\beta I_{\mathbf{k}_{1}}} \cdot \ldots \cdot \sqrt{\beta I_{\mathbf{k}_{n}}} \rho_{\mathbf{k}_{1}} \ldots\right. \\
& \left.\times \rho_{\mathbf{k}_{n}} \delta\left(\mathbf{k}_{1}+\ldots+\mathbf{k}_{n}\right) \partial^{n} / \partial(\beta h)^{n}\right) .
\end{aligned}
$$

Here Kronecker symbol means the irreducible sum of wave vectors $\mathbf{k}_{1}+\ldots+\mathbf{k}_{n}$, in which there are not subsets of vectors, the sum of which would be equalled to zero. The representation (2.8) determines 2-, 3- partial and higher orders of correlation in a system $[9,13]$. The restriction by a summand $\sim \frac{1}{N} \sum_{\mathbf{k} \neq 0} \beta I_{\mathbf{k}} \rho_{\mathbf{k}} \rho_{-\mathbf{k}}$ in an index of exponential function (2.8) is equivalent to a summation of a series, which was mentioned above. In this manner, we have a differential operator, which will act upon the energy of an ideal system

$$
\exp \left(\frac{1}{2 N} \sum_{\mathbf{k} \neq 0} \beta I_{\mathbf{k}} \rho_{\mathbf{k}} \rho_{-\mathbf{k}} \partial^{2} / \partial(\beta h)^{2}\right) \cdot F_{i d}\left(h_{\mathrm{M}}\right) .
$$

The differential operation can be exchanged by the integration

$$
\frac{1}{\sqrt{\pi}} \int_{-\infty}^{\infty} \mathrm{e}^{-\xi^{2}} F_{i d}\left[h_{\mathrm{M}}+2\left(\frac{1}{2 N} \sum_{\mathbf{k} \neq 0} \beta I_{\mathbf{k}} \rho_{\mathbf{k}} \rho_{-\mathbf{k}}\right)^{1 / 2} \xi\right] .
$$


As far as the dependence on variables $\rho_{\mathbf{k}}$ in (2.10) is a square-law, using the known methods $[14,15]$ one can reduce the integral in the Gaussian type. In a thermodynamic limit for the partition function of a system we shall get $Z=\mathrm{e}^{-\beta F\left(\bar{\rho}_{0}\right)}$, where

$$
F\left(\bar{\rho}_{0}\right)=\frac{N}{2} I_{0} \bar{\rho}_{0}^{2}+N \mathrm{e}^{\hat{D}} F_{i d}\left(\bar{h}_{\mathrm{M}}\right)-\frac{N}{\beta} \bar{\omega} \bar{S}+\frac{1}{2 \beta N} \sum_{\mathbf{k} \neq 0} \ln \left(1+\bar{\omega} \beta I_{\mathbf{k}}\right),
$$

and $\hat{D}=\bar{S} \partial^{2} / \partial(\beta h)^{2}$.

The parameters $\bar{\omega}, \bar{S}$ are determined from a condition of an extremum of the free energy (2.11)

$$
\partial F\left(\bar{\rho}_{0}\right) / \partial \bar{\omega}=0, \quad \partial F\left(\bar{\rho}_{0}\right) / \partial \bar{S}=0,
$$

which will lead to the equations

$$
\bar{\omega}=-\mathrm{e}^{\hat{D}} b^{(1)}, \quad \bar{S}=\frac{1}{2 N} \sum_{\mathbf{k} \neq 0} \frac{\beta I_{\mathbf{k}}}{1+\bar{\omega} \beta I_{\mathbf{k}}} .
$$

A relation will also be applied here which relates a derivative from the free energy to cumulants of an ideal system of spins $-\beta F_{i d}^{(n)}=b^{(n-1)}$, and $b^{(n)}$ - mean n-th derivative from Brillouin function $b$. We can get the equation for an order parameter, evaluating a partial derivative from $(2.11)$ on $\bar{\rho}_{0}$, and as the result:

$$
\bar{\rho}_{0}=\mathrm{e}^{\hat{D}} b\left(h_{\mathrm{M}}\right)=\frac{1}{\sqrt{\pi}} \int_{-\infty}^{\infty} \mathrm{e}^{-\xi^{2}} b\left(h_{\mathrm{M}}+2 \bar{S}^{1 / 2} \xi\right) .
$$

In this manner, we come to a known system of equations (2.12) and (2.13) [4-8], which will describe the behaviour of an order parameter in the two-tails approximation, where $\bar{S}$ determines a variance of Gaussian distribution of fluctuations of an order parameter. The formula (2.11) determines a free energy of a system in this approximation and was obtained in [4] by a method of diagram techniques.

We shall also specify the association with the exactly solved models of phase transition, which were determined at a phenomenological level (see $[15,16]$, and also review the publications, which are quoted there). A change from the formally exact expression (2.8) to the approximate (2.9) according to [15] means the change to an exactly solved model (for arbitrary dimensionality of space) in case of an Ising model. The exactly solved model introduced in this manner, is not absolutely satisfactory, since it, as it was indicated, will not describe the phase transition of the second order. This fact can also be interpreted in such a manner that the account of fluctuations is overstated, which will give rise to the fluctuation modification of an order of transition [15].

The analysis of the contributions of higher orders according to formulas (2.8) is quite a composite problem, as we have a functional integral of a non-Gaussian type. The analysis of the indicated contributions will be implemented by means of a variational principle that will be described in the following section. 


\section{Variational approach in the method of functional integration}

This method is grounded on the Bogolyubov inequality for the free energy of a system. In the method of functional integration, this inequality can be written as $[14,17,18]$

$$
F\left(\bar{\rho}_{0}\right) \leqslant F_{0}\left(\bar{\rho}_{0}\right)+\left\langle H(\{\rho\})-H_{0}(\{\rho\})\right\rangle_{0} .
$$

Here $H(\{\rho\})$ and $H_{0}(\{\rho\})$ respectively mean exact and trial Hamiltonians, which are functions of a variable integration. The angular brackets in (3.1) mean an average with the application of a trial Hamiltonian. In this manner, according to (2.5), (2.6) we can write

$$
H(\{\rho\})=\frac{N}{2} I_{0} \bar{\rho}_{0}^{2}+\frac{1}{2 \beta} \sum_{\mathbf{k} \neq 0} \rho_{\mathbf{k}} \rho_{-\mathbf{k}}+\sum_{i} F_{i d}\left(h_{\mathrm{M}}+h_{i}^{\prime}\right) .
$$

The trial Hamiltonian contains certain variation parameters, and is picked so that the average in (3.1) is equal to zero. The most prime form of a trial Hamiltonian corresponds to a random phase approximation

$$
H_{0}(\{\rho\})=N C\left(\bar{\rho}_{0}\right) / \beta+\frac{N}{2} I_{0} \bar{\rho}_{0}^{2}+\frac{1}{2 \beta} \sum_{\mathbf{k} \neq 0}\left(1-\beta \alpha_{\mathbf{k}}\right) \rho_{\mathbf{k}} \rho_{-\mathbf{k}}+N F_{i d}\left(h_{\mathrm{M}}\right) .
$$

The parameters $\alpha_{\mathbf{k}}$ have a sense of the Fourier-components for an effective interaction and can be obtained from the condition of the extremum of free energy. Therefore, for average quantities in (3.1) we shall get

$$
\left\langle H_{0}(\{\rho\})\right\rangle_{0}=N C\left(\bar{\rho}_{0}\right) / \beta+\frac{N}{2} I_{0} \bar{\rho}_{0}^{2}+N F_{i d}\left(h_{\mathrm{M}}\right)+\frac{1}{2 \beta} \sum_{\mathbf{k} \neq 0}\left(1-\beta \alpha_{\mathbf{k}}\right)\left\langle\rho_{\mathbf{k}} \rho_{-\mathbf{k}}\right\rangle_{G},
$$

where the calculation average for a variable $\rho_{\mathbf{k}}$ is executed on the basis of a Hamiltonian (3.3) and in particular $\left\langle\rho_{\mathbf{k}} \rho_{-\mathbf{k}}\right\rangle_{G}=1 /\left(1-\beta \alpha_{\mathbf{k}}\right)$. Based on (3.1)-(3.4) we can get the following expression for defining $C\left(\bar{\rho}_{0}\right)$

$$
C\left(\bar{\rho}_{0}\right) / \beta=-F_{i d}\left(h_{\mathrm{M}}\right)+\left\langle F_{i d}\left(h_{\mathrm{M}}+h_{i}\right)\right\rangle_{G}+\frac{1}{2 \beta N} \sum_{\mathbf{k} \neq 0} \beta \alpha_{\mathbf{k}} /\left(1-\beta \alpha_{\mathbf{k}}\right) .
$$

For the average in (3.5) we shall also get

$$
\left\langle F_{i d}\left(h_{\mathrm{M}}+h_{i}\right)\right\rangle_{G}=\mathrm{e}^{\hat{D}} F_{i d}\left(h_{\mathrm{M}}\right), \quad \hat{D}=\frac{1}{2 N} \sum_{\mathbf{k} \neq 0} \beta I_{\mathbf{k}} /\left(1-\beta \alpha_{\mathbf{k}}\right) \cdot \partial^{2} / \partial(\beta h)^{2} .
$$

In this manner, for the free energy of a zeroth-order approximation (3.3) we shall get

$$
F_{0}\left(\bar{\rho}_{0}\right)=\frac{N}{2} I_{0} \bar{\rho}_{0}^{2}+N \mathrm{e}^{\hat{D}} \cdot F_{i d}\left(h_{\mathrm{M}}\right)+\frac{1}{2 \beta} \sum_{\mathbf{k} \neq 0} \beta \alpha_{\mathbf{k}} /\left(1-\beta \alpha_{\mathbf{k}}\right)+\frac{1}{2 \beta} \sum_{\mathbf{k} \neq 0} \ln \left(1-\beta \alpha_{\mathbf{k}}\right)
$$


From the equation for parameters $\left(\partial F_{0}\left(\bar{\rho}_{0}\right) / \partial \alpha_{\mathbf{k}}=0\right)$ we shall get that $\alpha_{\mathbf{k}}=$ $I_{\mathbf{k}} \mathrm{e}^{\hat{D}} b^{(1)}\left(\bar{h}_{\mathrm{M}}\right)$. In this manner for defining $\alpha_{\mathbf{k}}$ it is necessary to solve the integral equation. The fitting condition suffices for a thermodynamic description

$$
\bar{S}=\frac{1}{2 N} \sum_{\mathbf{k} \neq 0} \beta I_{\mathbf{k}} /\left(1-\beta I_{\mathbf{k}} \mathrm{e}^{\hat{D}} b^{(1)}\left(\bar{h}_{\mathrm{M}}\right)\right),
$$

where $\hat{D}=\bar{S} \cdot \partial^{2} / \partial(\beta h)^{2}$.

Having calculated a partial derivative of the free energy (3.7) on $\bar{\rho}_{0}$, we shall get the equation for an order parameter. It is easy to see that these results coincide with (2.11)-(2.13). The examples considered above also demonstrate a corrigibility of the improvement of a two-tails approximation by a corresponding selection of a trial Hamiltonian. Applying the arguments stated in section 2 (formula (2.9)), we use the following structure of a trial Hamiltonian

$$
H_{0}(\{\rho\})=N C\left(\bar{\rho}_{0}\right) / \beta+\frac{N}{2} I_{0} \bar{\rho}_{0}^{2}+\frac{1}{2 \beta} \sum_{\mathbf{k} \neq 0} \rho_{\mathbf{k}} \rho_{-\mathbf{k}}+N \mathrm{e}^{\hat{D}_{1}(\rho)} F_{i d}\left(h_{\mathrm{M}}\right),
$$

where $\hat{D}_{1}(\rho)=\frac{1}{2 N} \sum_{\mathbf{k} \neq 0} \beta \alpha_{\mathbf{k}} \rho_{\mathbf{k}} \rho_{-\mathbf{k}} \cdot \partial^{2} / \partial(\beta h)^{2}$. Having produced the corresponding calculations we shall get the equation for $C\left(\bar{\rho}_{0}\right)$,

$$
C\left(\bar{\rho}_{0}\right) / \beta=\left(\left\langle\mathrm{e}^{\hat{D}_{2}(\rho)}\right\rangle_{0}-\left\langle\mathrm{e}^{\hat{D}_{1}(\rho)}\right\rangle_{0}\right) F_{i d}\left(h_{\mathrm{M}}\right)
$$

where the operator in (3.10) is determined by the formula

$$
\hat{D}_{2}(\rho)=\frac{1}{\sqrt{N}} \sum_{\mathbf{k} \neq 0} \sqrt{\beta I_{\mathbf{k}}} \mathrm{e}^{\mathbf{i k r}} \rho_{\mathbf{k}} \cdot \partial / \partial(\beta h) .
$$

In this manner, the contribution of higher correlations in the partition function (3.1) is executed on the average that is expressed by the formula (3.10). In a thermodynamic limit after some calculations, which we omit, we shall get

$$
C\left(\bar{\rho}_{0}\right) / \beta=\left(\mathrm{e}^{\hat{\bar{D}}_{2}}-\mathrm{e}^{\hat{\bar{D}}_{1}}\right) \cdot F_{i d}\left(h_{\mathrm{M}}\right)
$$

where

$$
\hat{\bar{D}}_{2}=\frac{1}{2 N} \sum_{\mathbf{k} \neq 0} \beta I_{\mathbf{k}} /\left(1+\beta \bar{\omega} \alpha_{\mathbf{k}}\right) \cdot \partial^{2} / \partial(\beta h)^{2}, \quad \bar{\omega}=-\mathrm{e}^{\hat{\bar{D}}_{1}} b^{(1)}\left(h_{\mathrm{M}}\right) .
$$

The free energy has the following structure

$$
F_{0}\left(\bar{\rho}_{0}\right)=N C\left(\bar{\rho}_{0}\right) / \beta+\frac{N}{2} I_{0} \bar{\rho}_{0}^{2}+N \mathrm{e}^{\hat{\bar{D}}_{1}} \cdot F_{i d}\left(\bar{h}_{\mathrm{M}}\right)-\frac{N}{\beta} \bar{\omega} \bar{S}_{1}+\frac{1}{2 \beta} \sum_{\mathbf{k} \neq 0} \ln \left(1+\bar{\omega} \beta \alpha_{\mathbf{k}}\right) \text {. }
$$

Based on (2.11), we can rewrite (3.12) in the form

$$
F_{0}\left(\bar{\rho}_{0}\right)=\frac{N}{2} I_{0} \bar{\rho}_{0}^{2}+N \mathrm{e}^{\hat{\bar{D}}_{2}} \cdot F_{i d}\left(\bar{h}_{\mathrm{M}}\right)-\frac{N}{\beta} \bar{\omega} \bar{S}_{1}+\frac{1}{2 \beta} \sum_{\mathbf{k} \neq 0} \ln \left(1+\bar{\omega} \beta \alpha_{\mathbf{k}}\right) .
$$


From the equation $\partial F_{0}\left(\bar{\rho}_{0}\right) / \partial \alpha_{\mathbf{k}}=0$ is determined $\alpha_{\mathbf{k}}$

$$
\bar{\omega} \alpha_{\mathbf{k}}=-\bar{b}^{(1)} I_{\mathbf{k}}-\tilde{b}^{(1)} \alpha(0),
$$

where

$$
\bar{b}^{(1)}=\mathrm{e}^{\hat{\bar{D}}_{2}} b^{(1)}\left(h_{\mathrm{M}}\right), \quad \tilde{b}^{(1)}=\mathrm{e}^{\hat{\bar{D}}_{1}} b^{(1)}\left(h_{\mathrm{M}}\right) .
$$

Here corresponding operators can also be written in the form

$$
\hat{\bar{D}}_{1}=\bar{S}_{1} \cdot \partial^{2} / \partial(\beta h)^{2}, \quad \hat{\bar{D}}_{2}=\bar{S}_{2} \cdot \partial^{2} / \partial(\beta h)^{2} .
$$

The structure of the free energy (3.13) corresponds to a two-tails approximation with the distinction that there are two functions here

$$
\bar{S}_{1}=\frac{1}{2 N} \sum_{\mathbf{k} \neq 0} \beta \alpha_{\mathbf{k}} /\left(1+\bar{\omega} \beta \alpha_{\mathbf{k}}\right), \quad \bar{S}_{2}=\frac{1}{2 N} \sum_{\mathbf{k} \neq 0} \beta I_{\mathbf{k}} /\left(1+\bar{\omega} \beta \alpha_{\mathbf{k}}\right) .
$$

The free component in (3.14) (which does not depend on a wave vector) can be written as follows

$$
\beta \tilde{b}^{(1)} \alpha(0)=\tilde{b}^{(3)} \frac{\bar{S}_{3} \cdot \bar{b}^{(1)} / \tilde{b}^{(1)}-\bar{S}_{4}}{1-\bar{S}_{4} \cdot \tilde{b}^{(3)}} .
$$

The equation (3.14) (taking into account (3.16)) is an integral equation for defining the effective interaction $\alpha_{\mathbf{k}}$. For describing the thermodynamics there are enough fitting conditions, which reduce, accordingly, in two equations for functions $\bar{S}_{3}, \bar{S}_{4}$

$$
\bar{S}_{3}=\frac{1}{2 N} \sum_{\mathbf{k} \neq 0} \beta I_{\mathbf{k}} \beta \alpha_{\mathbf{k}} /\left(1+\bar{\omega} \beta \alpha_{\mathbf{k}}\right)^{2}, \quad \bar{S}_{4}=\frac{1}{2 N} \sum_{\mathbf{k} \neq 0}\left(\beta \alpha_{\mathbf{k}}\right)^{2} /\left(1+\bar{\omega} \beta \alpha_{\mathbf{k}}\right)^{2} .
$$

We shall get the equation for an order parameter calculating a partial derivative from $(3.13)$ on $\bar{\rho}_{0}$, and as a result

$$
\bar{\rho}_{0}=\frac{1}{\sqrt{\pi}} \int_{-\infty}^{\infty} \mathrm{e}^{-\xi^{2} b}\left(h_{\mathrm{M}}+2 \bar{S}_{2}^{1 / 2} \xi\right) .
$$

The system of equations (3.14)-(3.18) generalizes a two-tails approximation in the above suggested method. The function $\bar{S}_{2}$ is an analogy of a two-tails approximation and determines a variance of Gaussian distribution of fluctuations of an order parameter. The system of equations (3.15), (3.17) and (3.18) is closed and allows us to determine temperature dependences of an order parameter and functions $\bar{S}_{1}, \bar{S}_{2}, \bar{S}_{3}, \bar{S}_{4}$.

Further on we shall be restricted only to a numerical analysis of the obtained system of equations. The calculations were executed for an Ising model of a spin $S=1 / 2$. In figure 1 the dependences of an order parameter $\bar{\rho}_{0}$ and $\bar{S}_{2}$ (curves 1 and 1 ', respectively) for a cubic lattice (coordination number $\mathrm{z}=6$ ) are reduced. Besides, the corresponding results for a two-tails approximation (curves 2 and 2') are reduced, as well as the temperature dependence of an order parameter of a mean field (curve $3)$. Temperature is presented in dimensionless units $\left(t=k_{\mathrm{B}} T / z I\right)$. We can see, 


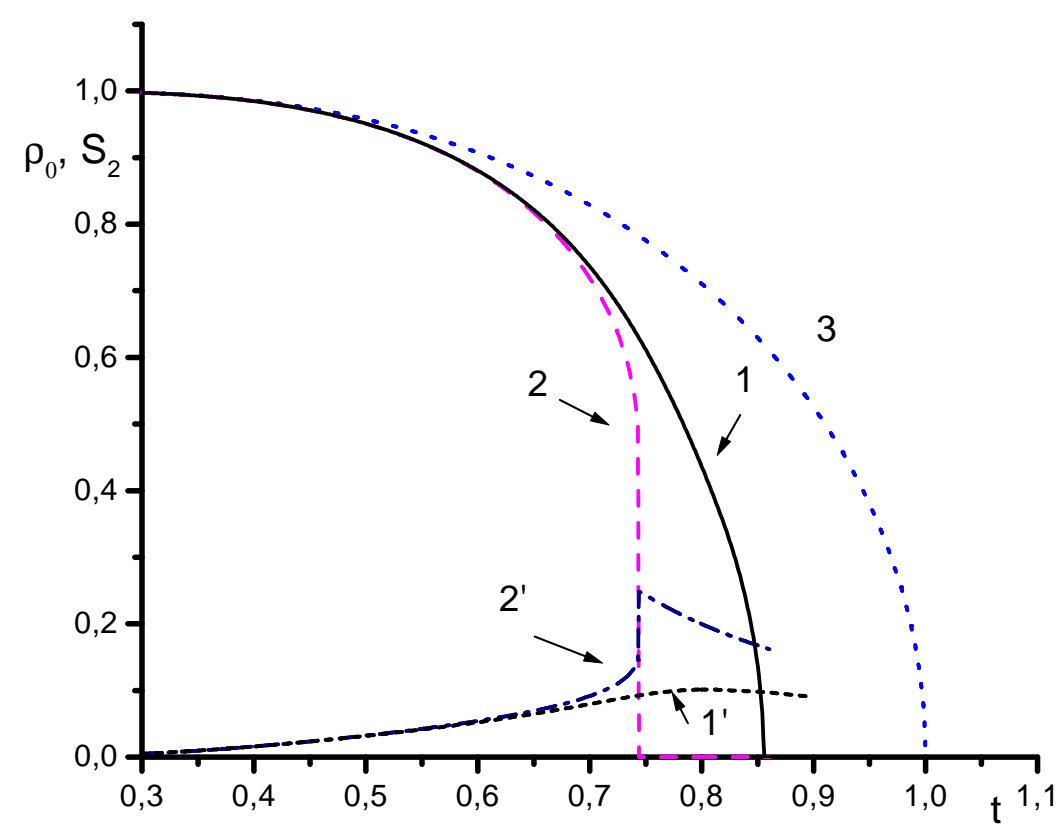

Figure 1. Temperature dependences of an order parameter and variance for a cubic lattice in the suggested approximation (curves 1 and 1', respectively); in a two-tails approximation (curves 2 and 2'); mean field - curve 3.

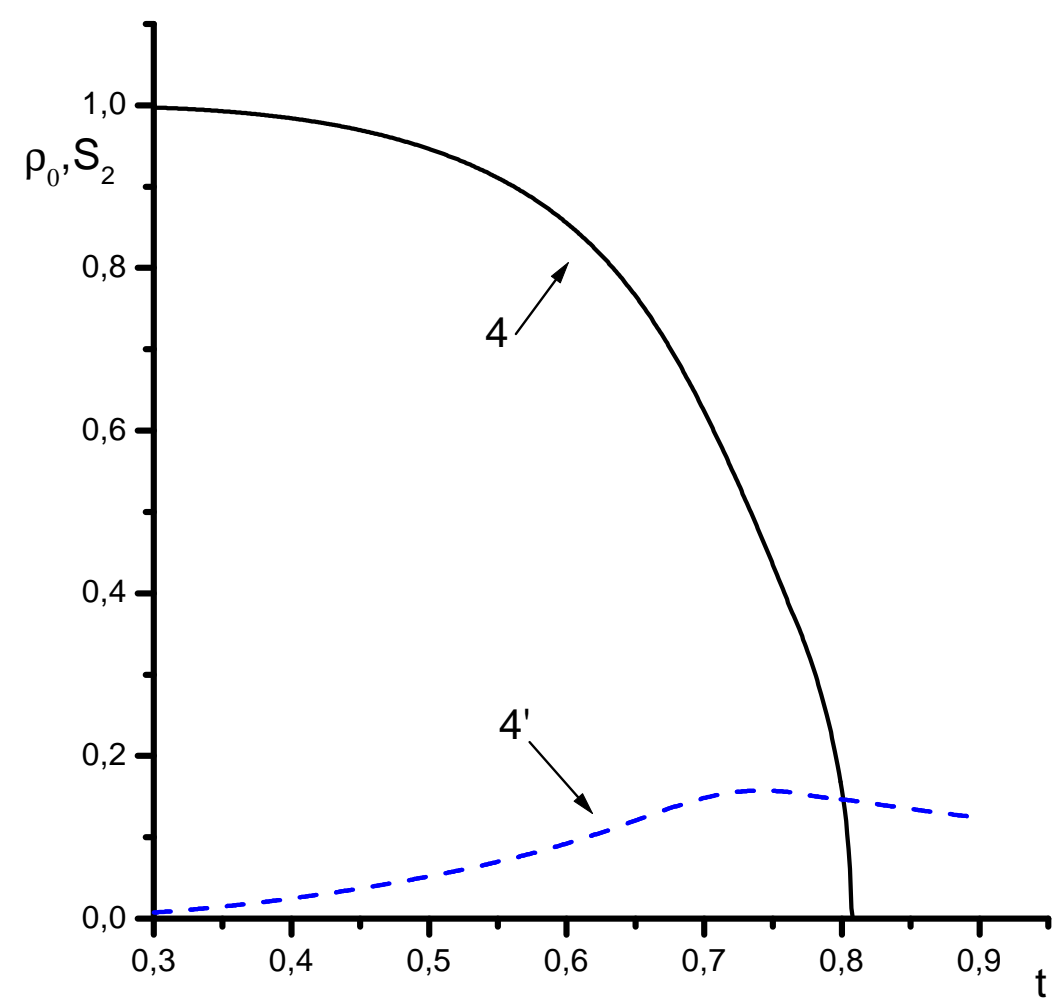

Figure 2. Temperature dependences of an order parameter (curve 4) and variance (curve 4') for a square lattice in the suggested approximation. 
that the suggested approximation will describe a continuous phase transition with a satisfactory magnitude of the temperature of transition $\left(t_{\mathrm{C}} \approx 0,86\right)$. The variance $\bar{S}_{2}$ is a monotonous function of temperature and will pass through maxima, some of which are in the region of low temperatures. Figure 2 illustrates the corresponding results for a two-dimensional case $(\mathrm{z}=4)$. It is seen that the maxima of a variance is higher (curve 4'), which is in accordance with the known fact of the more expressed fluctuation of an order parameter for a two-dimensional system. We also see a certain modification of curvature in the temperature dependence of order parameters in the field of joint low-temperature and critical solutions. The considered approximation gives a bit overestimated magnitude for the temperature of a phase transition $\left(t_{\mathrm{C}} \approx\right.$ 0,81 ) (we shall recall that an Onsager solution gives $t_{\mathrm{C}} \approx 0,57$ ). In this manner, the suggested approximation will also describe qualitatively the two-dimensional system, while in a two-tails approximation the solution for an order parameter is missing [8].

\section{Conclusions}

Summing up, we shall underline that in the present paper a rather simple method is presented which qualitatively correctly and quantitatively enough, in a self-consistent mode takes into account the fluctuations of an order parameter in the Ising model of phase transition. Certainly, any self-consistent method can not compete with the method of a renormalization group, which gives "precise" values of critical parameters, but can be applied only in a narrow neighbourhood of a critical point.

The choice of a functional integration method in our case is convenient as it allows us to formulate an approximation in the terms of functionals of the free energy and to immediately apply a variational principle. In this manner, in the present paper the functional of the free energy is determined which will reproduce the results of a two-tails approximation in a diagram method. On this basis, with the application of a variational principle, the self-consistent set of equations is obtained which will describe the fluctuations of an order parameter in the form of a Gaussian distribution. The method considered can also be easily generalized for the other models of phase transitions.

\section{Acknowledgements}

The author thanks I.V.Stasyuk and the collaborators of the department of quantum statistics for their critical and stimulating discussions.

\section{References}

1. Izyumov Yu.A., Kassan-Ogly F.A., Skriabin Yu.N. Field Method in the Ferromagnetism Theory. Moscow, Nauka, 1974 (in Russian). 
2. Izyumov Yu.A., Skriabin Yu.N. Statistical Mechanics of Magnetic-ordered Systems. Moscow, Nauka, 1987 (in Russian).

3. Bar'yakhtar V.G., Krivoruchko V.V., Yablonsky D.A. Green Functions in Theory of Magnetism. Kiev, Naukova Dumka, 1984 (in Russian).

4. Yukhnovskii I.R., Levitskii R.R., Sorokov S.I. Investigation of quasi-spin system in the basic approach. // Cond. Mat. Phys., 1993, vol. 1, p. 43-62.

5. Garanin D.A., Lutovinov V.S. Quasi-Taylor series in the theory of magnetism. // Theor. Math. Phys., 1985, vol. 62, No. 2, p. 263-271.

6. Onyszkiewicz Z., Wierzbicki A. Regularization of Feynman diagrams in the high density expansion method for spin systems near critical points. // Physics Letters A, 1986, vol. 116, No. 7, p. 335-339.

7. Onyszkiewicz Z., Wierzbicki A. // Physica B, 1986, vol. 151, p. 462.

8. Popov M.A. Gaussian approximation in the Ising model with long-range interaction. // Theor. Math. Phys., 1990, vol. 83, No. 3, p. 455-461.

9. Yukhnovskii I.R. Phase Transitions of the Second Order. Collective Variable Method. Kiev, Naukova Dumka, 1985 (in Russian).

10. Kozlovskii M.P., Yukhnovskii I.R. Order parameter and free energy in the threedimensional Ising model below the phase transition point. // Theor. Math. Phys., 1989, vol. 79, No. 1, p. 135-145 (in Russian).

11. Kozlovskii M.P., Pylyuk I.V. Investigation of the critical characteristics of the threedimensional Ising model using the non-Gaussian measure densities. // Ukr. Fiz. Zhurn., 1990, vol. 35, No. 1, p. 146-147 (in Russian).

12. Vakarchuk I.A., Rudavskii Yu.K. Functional integration method in the theory of the spin systems. // Theor. Math. Phys., 1981, vol. 49, No. 2, p. 234-247.

13. Yukhnovskii I.R., Holovko M.F. The Statistical Theory of Classical Equilibrium Systems. Kiev, Naukova Dumka, 1980 (in Russian).

14. Moriya T. Spin fluctuations in itinerant electron magnetism. Moscow, Mir, 1988 (in Russian) (in Russian).

15. Ivanchenko Yu.M., Filippov A.E., Radievskii A.V. Nonlinear excitations in the critical region. // Ukr. Fiz. Zhurn., 1993, vol. 38, No. 4, p. 607-615.

16. Ivanchenko Yu.M., Filippov A.E., Radievskii A.V. Phase transition in superconductive systems with d-coupling. // Fiz. Tv. Tela, 1989, vol. 31, No. 10, p. 204-213.

17. Ermilov A.N., Kireev A.N., Kurbatov A.M. Investigation of spin glass models with arbitrary distribution of interaction constants. // Theor. Math. Phys., 1982, vol. 49, No. 3, p. 344-352.

18. Gorshkovt S.N., Zabrodin A.V., Rodriges K., Fedianin V.K. On the Feynman variational principle for the polaron in magnetic field. // Theor. Math. Phys., 1985, vol. 62, No. 2, p. 304-311. 
Гаусове наближення в моделі Ізінга. Варіаційний метод

\section{В.С.Янішевський}

Дрогобицький педагогічний університет ім. І.Франка, Львівська обл., 293720 Дрогобич, вул. І.Франка, 24

Отримано 25 грудня 1998 р., в остаточному вигляді - 3 травня 1999 p.

Запропоновано варіант гаусового наближення в ізінгових моделях фазових переходів. В основу підходу покладено метод функціонального інтегрування в поєднанні із варіаційним методом. Наведено гамільтоніан (функціонал), що відтворює в методі функціонального інтегрування відомі результати наближення двохвосток отримані діаграмним методом. Застосуванням варіаційного методу усувається головний недолік наближення двохвосток - неправильний опис роду фазового переходу. Наводяться чисельні розрахунки для дво- і тривимірної граток.

Ключові слова: модель Ізінга, функціональне інтегрування, гаусове наближення

PACS: $75.10 . \mathrm{H}$ 